\title{
Development of Fuzzy Decision Support System (FDSS) Procedures to Best Select e-Learning Solution
}

\author{
Mohammad A. A. Alhawamdeh \\ Consulting Manager \\ Bisher and Kariman Consulting Est. \\ PO Box 840078 - Amman 11181 - Jordan
}

\begin{abstract}
In the recent days, technological systems were developed to be more intelligent for serving the users' needs. As the result, the developers used Artificial Intelligence (AI) methods for this purpose. In addition, Decision Support System (DSS) used to be guide for the decision makers in determining the correct decision taken in specific situation. Since, DSS technique is used in many different areas: learning, health, spatial data, etc. Hence, the proposed algorithm-based procedures/steps in this paper depends on considering all offered systems' criteria and matching these criteria with the requirements of the academic institutions which desire to purchase an e-Learning system. This paper supports the selection of e-Learning system, which is new argument that needs AI technique based on decisional tree techniques. Later, more optimization work will be introduced to modify the current methodology.
\end{abstract}

\section{Keywords}

Decision criteria; fuzzy DEMATEL; e-learning selection

\section{INTRODUCTION}

Selecting e-Learning system is not simple operation; it needs accurate determinations and considering all factors. In this paper, the researcher tries to highlight the way of selecting eLearning system by enhancing an algorithm for selecting the enterprise resource planning (ERP) software. The researcher selected the algorithm from the area of "selecting best ERP software", wherein the ERP product was selected according to specific standards: time, cost, quality, and usability. Also, the researcher referred to three articles [1] [2] [3]. The e-Learning system is considered a software product as ERP product, which was highlighted and discussed in many studies [1] [2] [4]. The researchers in previous articles [1] [3] [5] explained that an ERP product has several factors that must be considered by the buyers. Furthermore, these factors are commonly mentioned in many studies as: vendor information, cost, quality, usability, and portability.

Thus, these factors are accredited at this paper to help the academic institutions to select the suitable e-Learning system. These factors are necessary and they must be taken into consideration when purchasing e-Learning system. The proposed system later will be designed and programmed based on the new algorithm. The current available offered eLearning systems will be collected from their vendors (websites). The criteria of these e-Learning systems will be taken into the proposed system to determine the functionality, usability, accuracy, portability and cost. This stage has commercial dimension, because it is focuses on the vendor ability for supporting the product. If the financial situation of the vendor is not able to provide the software product in specific date, the academic institution will not deal with this vendor. Also, the academic institution in this case is a customer, so the institution is responsible for collecting enough information about the vendor in order to avoid the risks later on. The next stage highlights the importance of system cost, which is necessary factor for the customer. Some people claim that the high cost is combined with high quality [3]. However, the customer must determine the suitable cost according to the financial abilities. After that, functionality of the system must be considered. The quality affects the functionality of the system [6]. Thus, high quality system leads to high functionality.

Moreover, portability indicates the ability of the system to be compatible with several operating systems and hardware devices. Based on article [3], this novel integrated DSS will depend on fuzzy number for ranking the offered e-learning systems. As it was mentioned in [2], fuzzy numbers were used for giving evaluation to the offered e-learning systems. Based on the fuzzy numbers, selection rate will be determined, since selection rate is between 0 and 1, if selection rate reaches to 1 , the e-learning system will be selected, and otherwise, the system will be not selected.

\subsection{Why DSS for Selection Software Product?}

The benefits of the proposed system can be summarized in the following points:

1. This system analyzes the criteria of the offered elearning systems.

2. This system is able to understand and analyze the academic institution requirements.

3. This system matches between the criteria of the offered systems and the requirements of the academic institution to recommend the most suitable e-earning system.

4. This system avoids the customers from any risks in providing the e-learning system form the vendor.

1.2 DSS Techniques in e-Learning Products The proposed DSS system based-algorithm procedure is useful as it depends on using fuzzy numbers with criteria of the offered systems as well as the institution requirements. This algorithm provides robust results through analyzing the criteria of the offered e-Learning systems as software product. Furthermore, this algorithm matches the criteria of the offered systems with the institution requirements to make decision process more robust. Moreover, this algorithm takes into consideration the cost of offered e-Learning systems in order 
to match with the financial ability of the academic institution that wants to purchase the system.

This algorithm is generated by mixing three models mentioned in [1] [2] [3] as shown in figure 1. These models were used for choosing the suitable ERP products and software products. This research proposed algorithm-based procedures benefited from these three models in many aspects:

1. Determining the criteria of the offered products.

2. Determining the customer requirements (academic institution).

3. Employing the fuzzy numbers to rank the offered systems according to the institution requirements.
Furthermore, algorithm in [6] built on DSS techniques in selecting ERP product. Thus, the proposed algorithm will be more robust and more accurate because it benefited from previous algorithms and models.

\section{LITERATURE SURVEY}

DSS is considered as computer-based information system that provides business or organizational decision making activities. DSS's serve the management, operations, and planning levels of an organization and help for making decisions. On the other side, DSS systems are artificial intelligence-based algorithms for providing the appropriate decisions. These algorithms give the decision after studying and considering all the problem aspects. Three main steps must be followed in designing DSS systems: Intelligence, Design, and Determine. However, DSS systems facilitate choosing the suitable decisions [7].

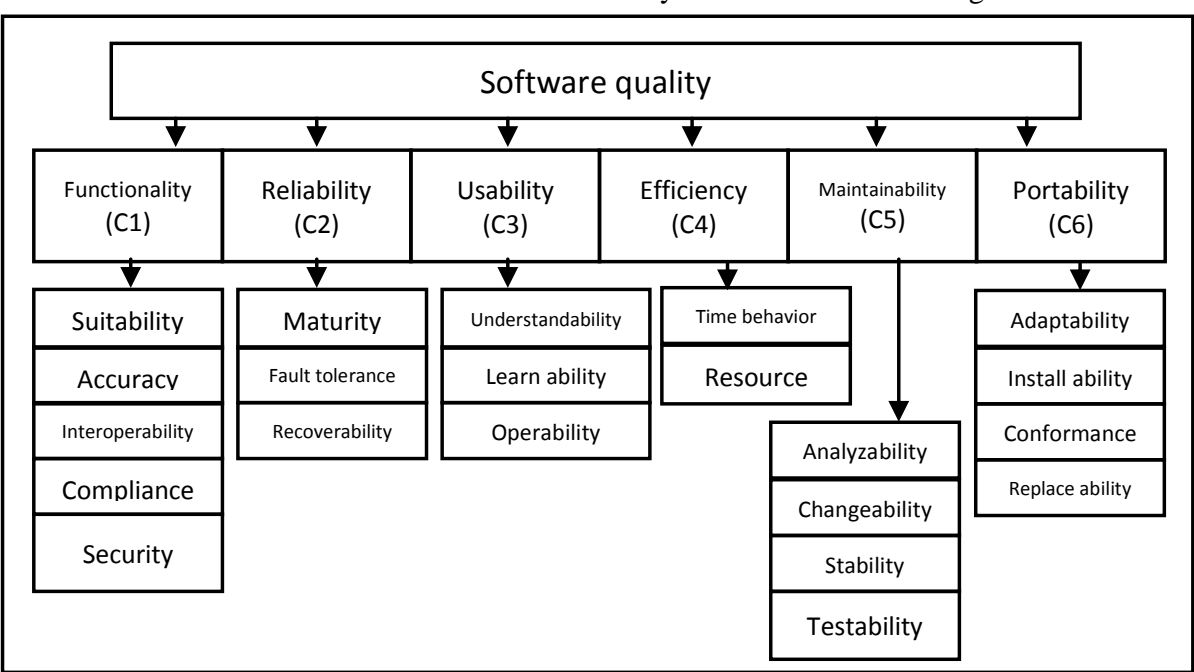

Framework for assessing the ERP system [3].

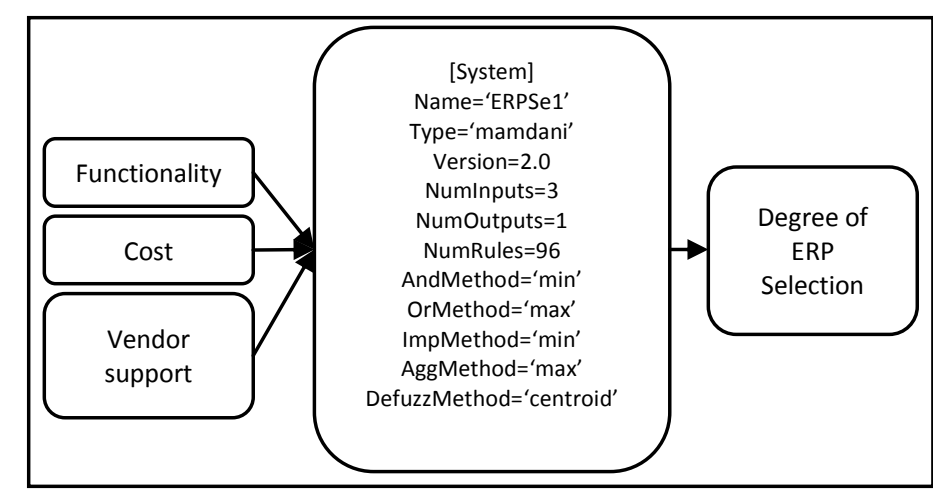

Proposed model for ERP selection base on fuzzy logic [2].
Evaluate and aggregate external professional data to obtain objective ERP suitability

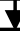

Combine evaluations of both data sources to obtain final fuzzy ERP suitability

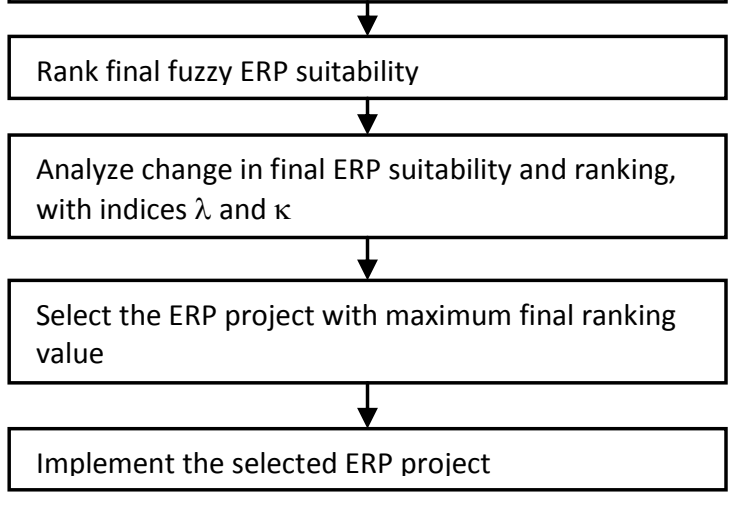

Algorithm of selecting ERP procedure [1].

Figure 1: The Proposed Algorithm-Based Procedures 
For academic institutions that want to move from the traditional learning to e-Learning, it is always challenging to choose the right learning system and the adequate technology to implement the suitable e-Learning solution. Since, eLearning systems that are adopted emanate in general from the need of institutions to integrate technologies for dispensing learning to their affiliates. Most adoptions have been driven in general by: I) the software solutions that are available on the market and the facilities, tools, and options they offer to learners and educators during the learning process and II) economic considerations [8]. Unfortunately, most of the academic institutions do not take into accounts some important factors in the decision making process towards purchasing an e-Learning system which frequently forces them to review their choices in a short period of time.

Engaging into e-Learning should not be restricted to the software system and its possibilities only but should take into account a set of criteria that play an essential role in creating an added value for selecting the most suitable an e-Learning to the institution [9]. These factors are: educational, technological, social and economic. Further, I) Educational: is related to the pedagogical methods used in teaching and learning [10]; II) Technological: considers the technologies used in the e-Learning system and how pedagogical principles are implemented with the available technologies; III) Engineering: focuses on the development of the e-Learning system through all the e-Learning system's life cycle; and IV) Socio-Economic: considers the feasibility of the system in terms of resources and its acceptance by a large community of users as well as the institutional budgets. The two latter aspects are practically ignored by most of the instructors and their assessments' protocols as they have minor impact on the learning process. Moreover, e-Learning systems need to be considered inside their real-life environment from the very early development phases where assessment focuses on design criteria and economic feasibility, till the late phases of operation since assessment focuses on performance and user satisfaction. They are conditioned in turn by a set of criteria among which the following I) the institution and the nature of its business, II) the type of learning that the institution would like to dispense to learners, III) the end users who are the learners and educators, IV) the stakeholders and the strategies they are planning to implement, V) the LMS (Learning Management System) to use in order to implement learning in the institution, and VI) the technologies used for learning. Obviously, these are not the only criteria. However, these are among the most important.

\section{1 e-Learning System's Aspects}

Designing e-learning system needs to take into considerations the motivation and quality of learning, time of response, and mobility which aims to make e-Learning system compatible with smart phones, tablets, etc.

\subsubsection{Design}

In this aspect, the e-Learning systems are designed according to high quality, which aids in obtaining good and robust learning process. According to [11], the communication tool in the e-Learning platform is necessary for achieving high quality in the education process. Also, the researcher introduced a case study of eduBITE, which consists of multimedia learning objects that are organized in a repository. Besides that, the researcher assured that to guarantee high quality and re-usability of learning objects the application of an appropriate development process for e-content development is required. However, courseware is considered as most important part of the e-Learning software. Designing the e-Learning platform must be responding to the learners' requirements and needs. [11] Highlighted that when designing the e-Learning platform, the developers must take into consider the instructor needs, learners' requirements, and the institution criteria that would use the e-Learning platform.

On the other side, [11] pore in studying the design of eduBITE platform and the researcher analyzed the system contents and relationships between the contents. The researcher analyzed the contents during the analysis phase. The analysis phase consists on analyzing learning objects and course structures in eduBITE, and then the researcher showed these contents in figure 2. Also, the researcher indicated that the learning objects have objectives producing competences for the learner. 


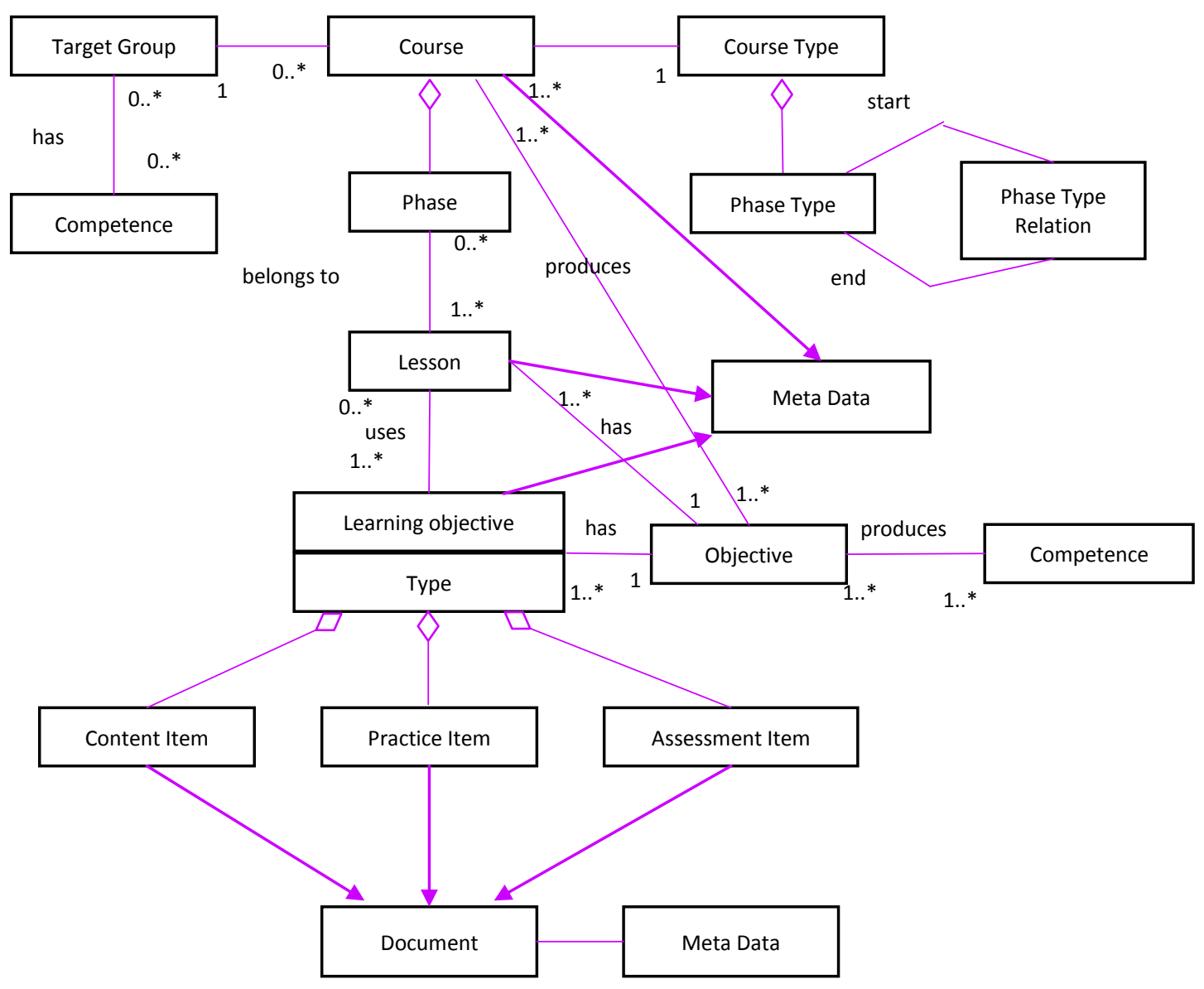

Figure 2: Objects of eduBITE

[12] Introduced a case study for Universal AV Services, a company that provides rental equipment and technical support for events. The researchers highlighted that using DSS systems for improving the company performance according to time, quality, and price. The researchers claimed that in the beginning of designing the system, it is important to determine how the equipment can fulfill the customer requirements. Also, they mentioned that the equipment rental problem focuses on yield management. In this research, a way of increasing the rental inventory is considered by "subhiring" from other suppliers. Figure 3 shows the proposed system architecture, which contains three main models: purchasing model, forecasting model, and data converter. Also, the system contains three main portions: user interface, system output, and original data. The proposed plan in this research was summarized in the following steps:

- Designing an algorithm for determining whether each rental item should be owned or sub-hired.

- Determining the total number of items for an equipment that should be purchased

- $\quad$ Finding the most appropriate forecasting method for this solution

- Designing an algorithm for transforming collected daily series into half-year usage.

For achieving this methodology, the researchers used MatLab for evaluating error performance. The forecasting model and data converter are developed in MatLab and integrated with

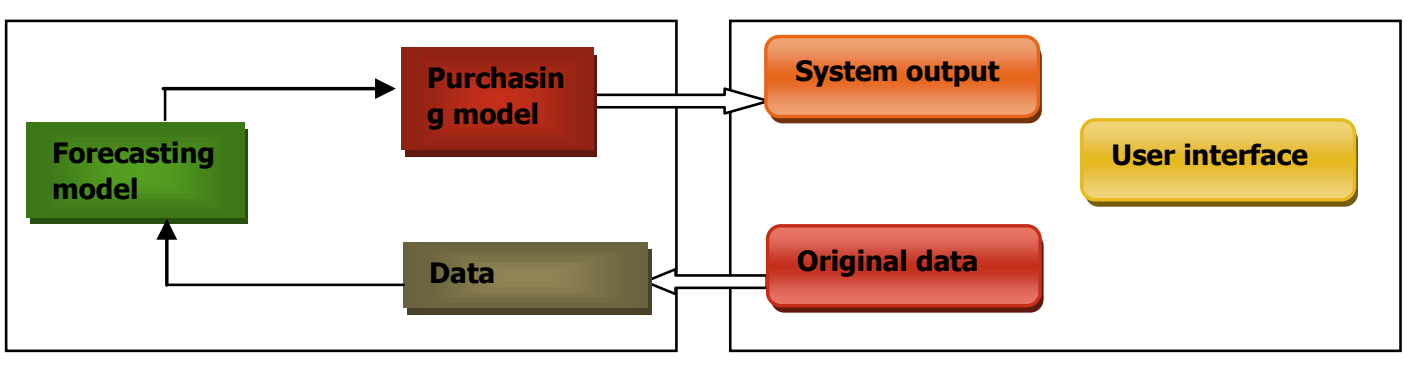

Figure 3: System Architecture 
the purchasing model developed in C\#.Net.

The researchers selected laptops as a research object. The collected equipment data includes both company owned laptops on hire and laptops sub-hired from suppliers. Furthermore, sub-hires explain the situation that equipment is in shortfall, which is critical for making purchasing. The researchers recorded the data for laptop in previous years in three ways: "namely equipment logbook on paper, job records, and usage history in an information system". Also, the daily data are input into a spreadsheet from the logbook by setting " 1 " for an item in use and " 0 " for idle item. After that, obtain a data set on laptop usage for the information system stage

The second stage was data converter, in which the required inputs for the system are peak equipment usages. The researchers used annual peak number of an equipment type in use $\mathrm{N}$ peak and the number of annual usage days Di for the number of item being greater than i. On the other hand, the researchers used a forecasting model. This model is divided into data usage and error measurement, and simulation on half-year usage. In the first section of model, the solution was introduced for obtaining internet access usage by the sum of actual data and forecasted data. In the other section, the researchers used exponential smoothing (ES) as a forecasting method. However, purchasing decisions address two problems: whether to own or to sub-hire for one item given the forecast peak demand and the number of a type of equipment, which should to be owned to minimize costs. The experiments compared forecasting methods by using annual usage data are carried out for comparing the characteristics of a number of forecasting methods for the research problem

\subsubsection{Integrity}

The e-learning of any system must be integrated with the current operating systems. This property enables the learner to use the e-Learning platform using any operating system whatever the tools are used [13]. Classified integration as a third level of importance, which brings to the offering of a hosting and cloud service. Also, the researcher highlighted that at the same level, the customers put technical features and quality of the learning object [14] indicated that the eLearning system must be integrated with the learner needs and instructor requirements. Each leaner study in different way and each instructor have special way in teaching the students, so the e-Learning system should be integrated with these needs to be flexible.

\subsubsection{Flexibility}

These criteria are considered as one of most difficult challenge that experiences the developers of e-Learning systems. Flexibility needs the system to be usable for the learner and instructor at the same time. As there are different learners' requirements, different instructors' learning strategies, and different operating systems used for using eLearning system. However, the system must be flexible in serving these types of users and it must be integrated with the various types of operating systems [15].

\subsubsection{Content Management System}

e-Learning website is multifunctional website which contains many contents, because e-Learning system must contain all functions of learning and education procedures. According to [16] each group of functions is located under main title, which expresses the role of the group. The groups of contents are managed in a way that expresses about the functions of the group. Many websites and systems use content management system but they employ it in inappropriate way, which will cause problems for the users when they use these systems. Most of e-Learning contents are functional contents, which execute services and functions. Not all learners are experts with websites particularly knowledge exchange via electronic tools. For this reason, it has to take into account the how the learner will behave when using the e-Learning system [17].

\subsubsection{Crowd Sourcing-Quality Control}

In the last decade, online learning had significant importance because of its role in developing the learning process. MOOC (Massive Open Online Courses) has shown great interest inspired by online courses by universities and tutors [18]. In the context of MOOC, e-Learning has been extended by making courses available online for anyone to participate for education purpose or self-learning interest. To assist the swift growth of online courses, crowd sourcing is considered in the form of peer-to-peer ( $p 2 p)$ or peer-to-crowd to provide support to leaners [19]. One of the major challenges in this context is quality control considering the fact that MOOC allows student to demonstrate their work on web in order to get feedback from a wide range of audiences (crowd source) to improve work. However, it cannot be expected that feedback will always be from experts as demonstrated work can divert to wide range of non-expert audience. Therefore, it requires significant attention to enhance the quality of learning experience by understating the crowd sourcing and its impact on learners. Furthermore, the Quality Control in MOOC platforms is important aspect and affected by technical problems such as traffic and user interfacing. In case of crowd sourcing, the problem of traffic and user interfacing will be more complicated because of the massive number of users that access the MOOC platform at the same time. If user interfacing is good and suitable for users, many users will use the system and the problem of traffic will appear. User interfacing can attract users when it is suitable and facilitate using platform but this case may generate traffic problem on MOOC system. Also, using multimedia tools in websites form traffic on the performance of the website because multimedia needs high processing "run at server" as most programming languages require. This problem may be more difficult if there are massive users execute this technique simultaneously. Also, this problem in general because of the MOOC itself has built based on insufficient analysis to be managed.

\subsection{Institution's Aspect}

Academic institutions have commercial interest with $\mathrm{e}$ Learning systems. So, the price factor is considered in the selecting procedure. Also, quality is considered as main criteria of e-Learning system. Academic institutions are important actors towards an effective implementation of eLearning system. It is fundamental responsibility of academic institutions to understand and implement critical design factors of e-learning system by identifying market demand, rules and policies, instructors' and learners' perspective, attitude, behavior, training and their vision towards system. [20] Suggest an advance e-Learning system should demonstrate certain features such as personalization, adaptability, extensibility, flexibility, mobility, interoperability for an effective system.

\subsubsection{Price}

In fact, the price of e-Learning system is important factor for the institution as any other system. Institution which interests with quality of the system does not care that much in the 
system's price, as the institution which interests with price does not care with quality that much as well, especially if the institution suffers from financial inability. According to [13], selecting e-Learning system is a complex task that involves different levels of the company and it is different from case to case. The researcher collected data context with quality and price aspects from many several companies, which use. The results showed that competitive price is a very important variable, which reached to $66 \%$ of company interest towards to e-Learning system.

Some providers sell the e-Learning system to institutions as open source in which the institution can update and modify the e-Learning platform according to its criteria "customization". On the contrary, some providers sell the systems to institution as a product, which cannot be updated, since the providers are not responsible for customizing the system. In the first case, the system price is considered as high because of its responsibility, but in the other case, the price is almost low. However, the institution is responsible for determining the type of the e-Learning system wants to purchase and the institution is responsible for the quality of the system, which is related with high price.

\subsubsection{Quality}

Quality is one of the important criteria for both the learners and institutions, since the institution takes into account the price besides to the quality and these two variables need deep thinking and to make decisions. On the other hand, the instructors interest with the technical aspects that enables introducing best learning process. According to [13], the quality is the first level when designing an e-Learning system, which is interested by the institutions. The researcher conducted a study on some academic institutions to view their needs when they select e-Learning system. However, the researcher found that $63 \%$ of the institution's interest is the quality of the e-Learning system.

\subsubsection{Time}

Feedback process is important factor of the e-Learning system for the learner and the instructor. This process depends on the system design in which the developers use robust methods and techniques that make feedback fast and accurate. Real time feedback has significant effect on the learner loyalty towards to e-Learning system. This effect comes from the time responding when the learner accesses a service. Like any other technical systems, e-Learning systems' developers care with response time.

\subsubsection{Integrity and Security}

The term of integrity in information technology refers to maintaining and assuring the accuracy and consistency of data over its entire lifecycle. Also, integrity is necessary property for databases and data storages. According to [21] integrity of data means that the data in database is reliable and accurate. On other hand, data warehousing and business intelligence control the accuracy of data in the databases and in warehouses. Data that has integrity is identically maintained during any operation, such as transfer, storage or retrieval. However, all data features such as: business rules, rules for how pieces of data related, dates, definitions and lineage must be correct for its data integrity to be complete. When executing functions on the data, the functions must ensure integrity. For examples: transferring the data, storing history and storing metadata.

\subsubsection{Adaptive e-Learning / User Profiles}

Adaptive learning is an important field in order to understand the learner's ability. Therefore, it is very critical for institutions to ignore adaptive learning in Learning Management Systems (LMSs). To offer a suitable learning path and learning resources to learners/students based on their interest and profiles, it is important to enrich learning resources and construct e-Leaning system more beneficial to not only attract more learners, but also get most out of the eLearning paradigm. In [22] study, multi-agent e-Learning system is prose since Item Response Theory (IRT) and Artificial Neural Networks (ANN) are considered to present adaptive tests and personalization. The agents using ANN can diagnose the leaner's learning behavior and their weaknesses in order to recommend the appropriate learning materials. In [23] an adaptive e-Learner system have been developed based on ontology approach to design semantic content, models to alter teaching process as per leaner characteristics, style, profile, preference, and knowledge levels. Based on ontology approach, domain model (to define domain topics and relationship using semantic), learner model (to define learner's characteristics) and content model (to define course and component structure) have been developed where regular tests have been used to understand learner's response using IRT to evaluate learner's ability. The construction of tests to understand and analyze personalization and adoption of learning material and environment and to evaluate learners play a critical role in adaptive e-Learning systems as these are responsible for high efficiency and reliability in e-Learning systems. With the transformation of web, the integration of reliable, computationally efficient, scalable adoption approaches has been great interest and demand by institutions to improve e-Learning systems.

\subsection{Learner's Aspect}

According to [24], in any learning environment, instructors are the principle players whereas learners are the primary participants. Both of these players and participants play a crucial role as their motivation and attitude has a strong impact on teaching and learning transactions.

\subsubsection{Training}

Some learners use traditional learning ways and others use developed learning ways. The institution should take into consideration the learning type. The integration of new technologies not only raises the challenges in hardware context but also software complexities. It raises the concerns to enhance the learner's technological skills using adaptability and usability by scaling the system complexity as per learner's requirement [25]. In addition, the use of chat applications, virtual help centres, forums and discussion forums have been used to office support. It is necessary to involve training plan in the e-learning system to be hint for the learners about using this type of learning (developed learning process).

\subsubsection{Mobility}

e-Learning platform as any other platforms must be compatible with more than one environment such as, Windows, Android, Linux, etc. The rapid growth of smart phones and hand held devices have changed have transformed the learning as an anytime anywhere learning. To exploit this, mobility in e-Learning paradigm has become a critical factor for its successful adoption. [26] Discussed the e-Learning in the context of m-Learning by exploiting mobile learning module of Moodle (MLE-Moodle) to analyze the learning and technological perspective. 


\subsubsection{Technology Acceptance}

Over the years, to securitize the influencing factors toward the adoption of technology, various technological adoption models are considered such as Technology Acceptance Model (TAM), Theory of Planned Behavior (TPB) [27], Unified Theory of Acceptance and Use of Technology (UTAUT) [28] and Theory of Reasoned Action (TRA) [29]. TAM model is extended by adding availability of conditions, training, workshop and technical help to understand the influence. Based on statistical analysis using survey data from various universities, it is identified that perceived ease of use and perceived usefulness are critical towards the adoption of eLearning. In [30] study, TAM is employed and identified that positive perception of technology effectiveness is very critical for students therefore associating it with student's attitude will help to raise the importance of e-Learning. In addition, other influencing factors such as performance expectancy, effort expectancy, and social influence, behavioral intention, and demographic (i.e. age, Gender and experience) can be considered to understand learners as well as instructors attitude towards the adoption of e-Learning technology.

\subsubsection{Graphical User Interface}

According to [31] the term of GUI covers all aspects of interaction between the user and the system. Each system must have good interface that enables user to interact with the system particularly learning system such as e-Learning systems. The system developer has to draw a plan of interaction the system via User Interface. Therefore, the developers must be careful when distribute buttons, textboxes, labels, etc. Also, the developers are responsible for coordinating the colors of forms and backgrounds. Moreover, some developers may focus on programming or security more than focusing on interfacing aspect. Hence, bad interface may cause problems in interaction with the system. When the user is not comfort with system interface, the user will behave wrongly with the system that will prevent user from arriving to the desired goal of accessing the system and will give bad impression to the user. Bad content management in the website may cause several difficulties to the user. To build good and strong websites in all aspects in either designing or security, the programmers use "Three Tier Technique": User Interface, Security Tier and Intermediate Tier. However, User Interface is considered as one of most important part of website designing procedure.

\subsection{Instructor's Aspect}

Instructor is the main component in the learning process and thus in e-learning. So, the developers of e-Learning platform must take into considerations the instructor interests and requirements such as, evaluation, education process, communication tools, feedback features (interaction with the students), etc.

\subsubsection{Motivation}

According to [32], the lack of motivation has been of great challenge from trainer's perspective. In these days, there is need to learn the new curricula particularly e-courses [32]. Disinclination on instructor's share to divert to e-Learning, exploiting their efforts for learners to access, lack of understanding of LMS enhances trouble for Higher Education institutes [33]. Also, it is necessary to promote the e-Learning programs by the academic institutions. The institution is responsible for attracting the instructors on benefits and develops an effective communication process to let end users to understand changes in an appropriate manner. On the other hand, the institution's management has ability to change learners and instructors motivation towards to the e-Learning systems. This can be done by advertisements, conferences, and workshops.

\subsubsection{Assessment}

Assessment is necessary operation for the instructor to evaluate the students using activities and virtual exams. The instructor draws the plan of course which contains activities and exams. Instructors need automated tools for the assessment process, which is mission of the system. However, assessment is a process to review all activities that instructors exploit to help and guide learners and to measure the learning progress and consequences of an individual in terms of knowledge, skills, and attitudes [34]. To reduce the time consumption on manual assessment and providing students a quicker feedback, enhancing assessment process within LMSs has been of great interest of institutes. Various centralized learning environments such as open source Moodle (http://moodle.org), Learn, Research, Network (http://dotlln.org/), and Blackboard (http://black board.com) in the form of LMSs has been mainly designed for course management, grading, classes and learning material. In addition to student's assessment using questionnaire, multiplechoice questions, uploading files. There is a lack of automated testing of various complex assignments such as software programming [35]. The integration of assessment for variety of subjects and patterns will provide several advantages to instructors. However, approaches to assess with reliability, high accuracy and appropriate feedback demand complex and efficient algorithms to reduce the human factor in assessment and examination process.

\subsubsection{Hinting}

In the context of instructing and learning, the hinting parameter is critical as it can be considered as a provision of some activities that might help learners to approach a solution of a problem [36]. To understand the hitting in the context of e-Learning and its comparison with human hitting, various studies and tutors have been developed such as Andes [37], SIETTE [38], AgentX [39]. In Munoz-Merino et al. study [36], based on experimental results it is discussed that hinting e-Learning system can replace the human teachers with same level of effectiveness. The study has been conducted without adoption of selecting different hinting techniques or learning process and therefore it can be enhanced through adaption process. An efficient hitting system can be very helpful for students to enhance learning whereas instruction can exploit it for assessment.

\subsubsection{Curricula}

Curriculum is a challenge that faces the instructors in the eLearning systems. This challenge increases in the case when the instructor is forced to keep curriculum up-to-date. Besides that, e-Learning has modernized and globalized educational activities without geographic limits. The cross-cultural learning environment has raised new challenges for the development of curriculum as curriculum demands expansion to meet global cultural aspects [40]. The adaptive learning has strong influence on e-Learning and it is important for instructors to analyze learners learning behavior and offer learners informative material best appropriate to their individual learning profile. In order to produce e-Learning more effective and productive, there needs to be some significant considerations to manage curriculum and cultural challenges. It adds additional burden on instructors to learn 
and keep up to date information, learn technologies, amend curriculum with appropriate material and resources to fulfill the learner's cultural needs.

\section{THE PROPOSED METHODOLOGY}

The e-Learning system is considered a software product as ERP product, which was highlighted and discussed in many previous studies. The researchers in articles [1] [3] [5], explained that an ERP product has several factors that must be considered by the buyers. Furthermore, the researcher mixed benefit parts from previous algorithm, framework, and model mentioned in articles [1] [2] [3].

The proposed algorithm consists of nine procedures/stages as shown in figure 4 . The first stage aims to collect information about the system vendor for avoiding any risk when providing the required software. Then, the institution determines the cost of the offered systems. After that, functionality is determined. This stage consist set of attributes that bear on the existence of a set of functions and their specified properties. Suitability, accuracy, and security are attributes of the functionality. Reliability is important stage which is consisted from set of attributes that bear on the capability of software to maintain its level of performance. The next stage is usability determination, which consists of a set of attributes that bear the effort needed for use and on the individual assessment of such use.

Also, portability has a set of attributes that bear on the ability of the software (e-Learning system) to be transferred from one environment to other one. Portability depends on: Install ability, Replacing ability and Adaptability as it was mentioned by [4]. However, these attributes will be taken into consideration. After these stages, the fuzzy numbers will be used to rank the offered e-Learning systems according to selection variable. As all technical systems, this algorithm must be involved in system that has user interface. The system matches between e-Learning systems and academic institution using questions appeared on pages (user interface).

The proposed procedures provide intelligent selection operation. This intelligence depends on the factors that are taken into considerations and it depends on the changes in the "selection procedure". Thus, the proposed algorithm makes the proposed system more robust and more accurate. The academic institutions are responsible to collect information about the e-Learning systems' vendors before beginning with selecting e-Learning system. The questions that will be asked from the system should reflect the details from the customer about the institution situation and requirements. 


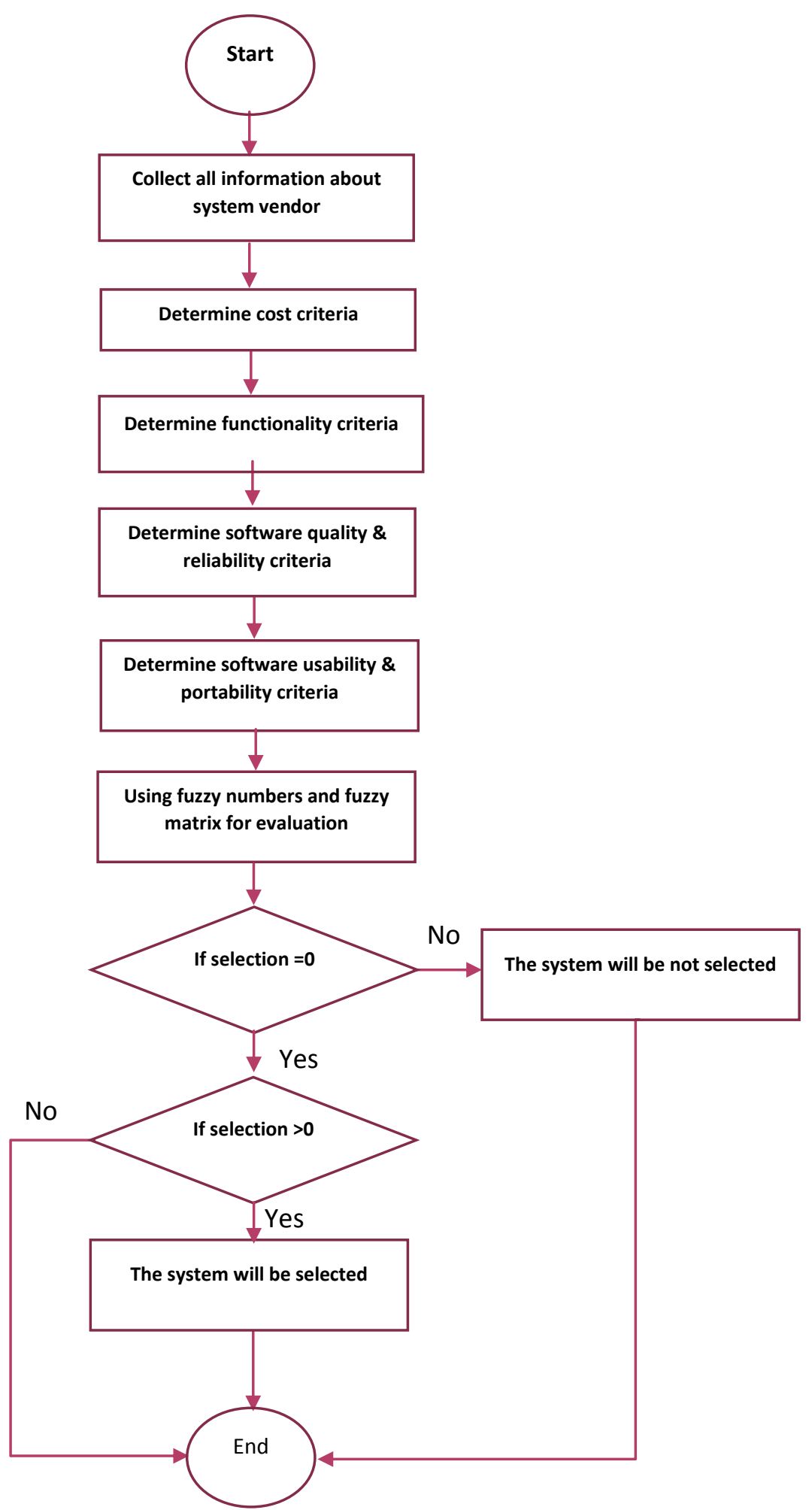

Figure 4: The Proposed Steps for Selecting e-Learning System 
Decision tree will be trained with the system criteria (quality) and vendor criteria. System criteria include functionality, portability, and cost. The problem of deciding the suitable elearning system is matching between the offered system
This equation gives the rate of each offered e-learning system to be used as fuzzy numbers. Also, the advantage of using this equation that is can prevent any extreme attribute value after transformation. The following figure 5 shows the weight of

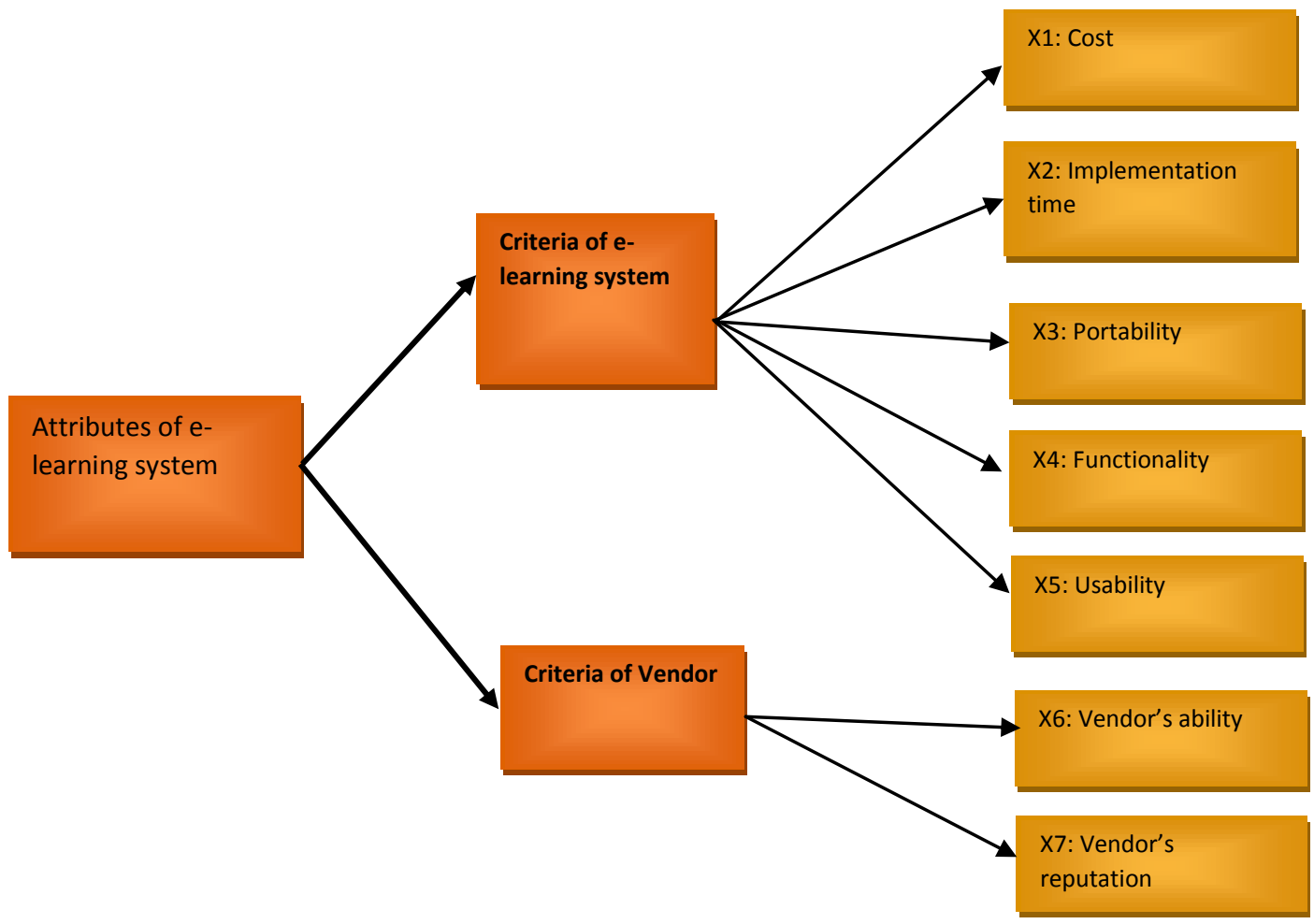

Figure 5: The Weights of Criteria

criteria and the academic institution requirements. The following decision tree aims to determine the best e-learning system.

The attributes of the e-learning systems are defined as $x_{i}(i=$ $1,2, \ldots, n)$. The attributes (criteria) weights are as $\mathrm{W}=\{\mathrm{VP}, \mathrm{P}$, F, G, VG\} [3], whereas VP: very poor, P: poor, F: fair, G: good, and VG: very good. These variables are used to give fuzzy numbers (rates). After that, define two variables (A, B) to be the set of benefit attributes and cost attributes. Let $T_{t i}(t=1,2, \ldots, m, i=1,2,3)$ represent the values assigned to e-learning system $E_{t}$.

$$
\text { Thus, } R T_{t i}=\frac{T_{t i}}{\sum_{t=1}^{m} T_{t i}}
$$

each attribute of the e-learning system.

The following algorithm that is indicated in figure 6 shows the steps followed in selectng an ERP product. This algorithm was mentioned in [41] that aimed to facilitiate selecting ERP product by the customers. In this paper, the researcher relied on this algorithm in the new procedure sof selecting e-learning system. the following algorithm depends on interviews condcuted with the instiutions and the vendors. However, figure 7 shows the echnacmenst on algorithm indicated in figure 6 . 


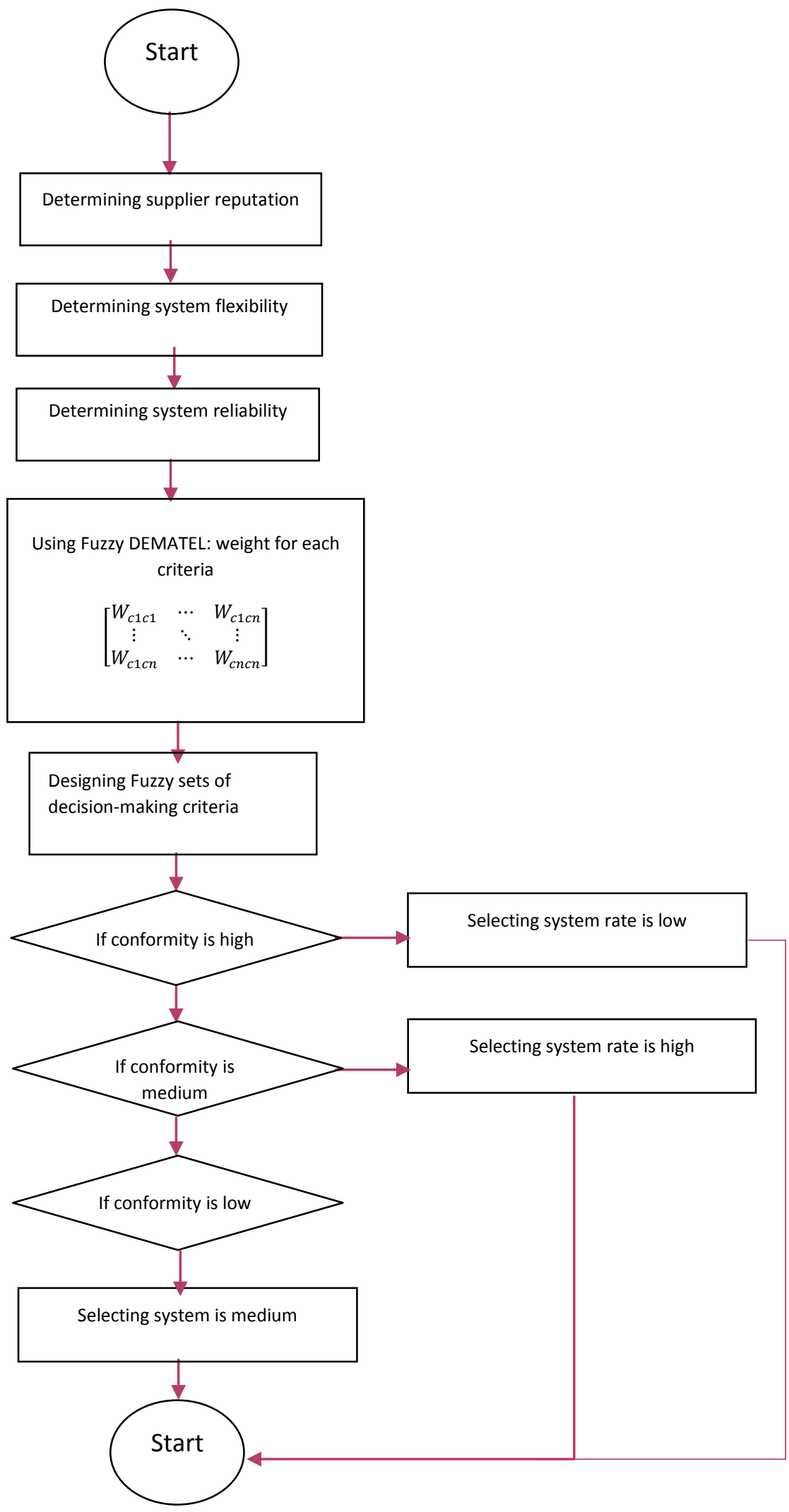

Figure 6: Selecting ERP Product Based on Heuristic Algorithm [41]. 
The enhanced proposed algorithm-based procedures as shown in figure 7:

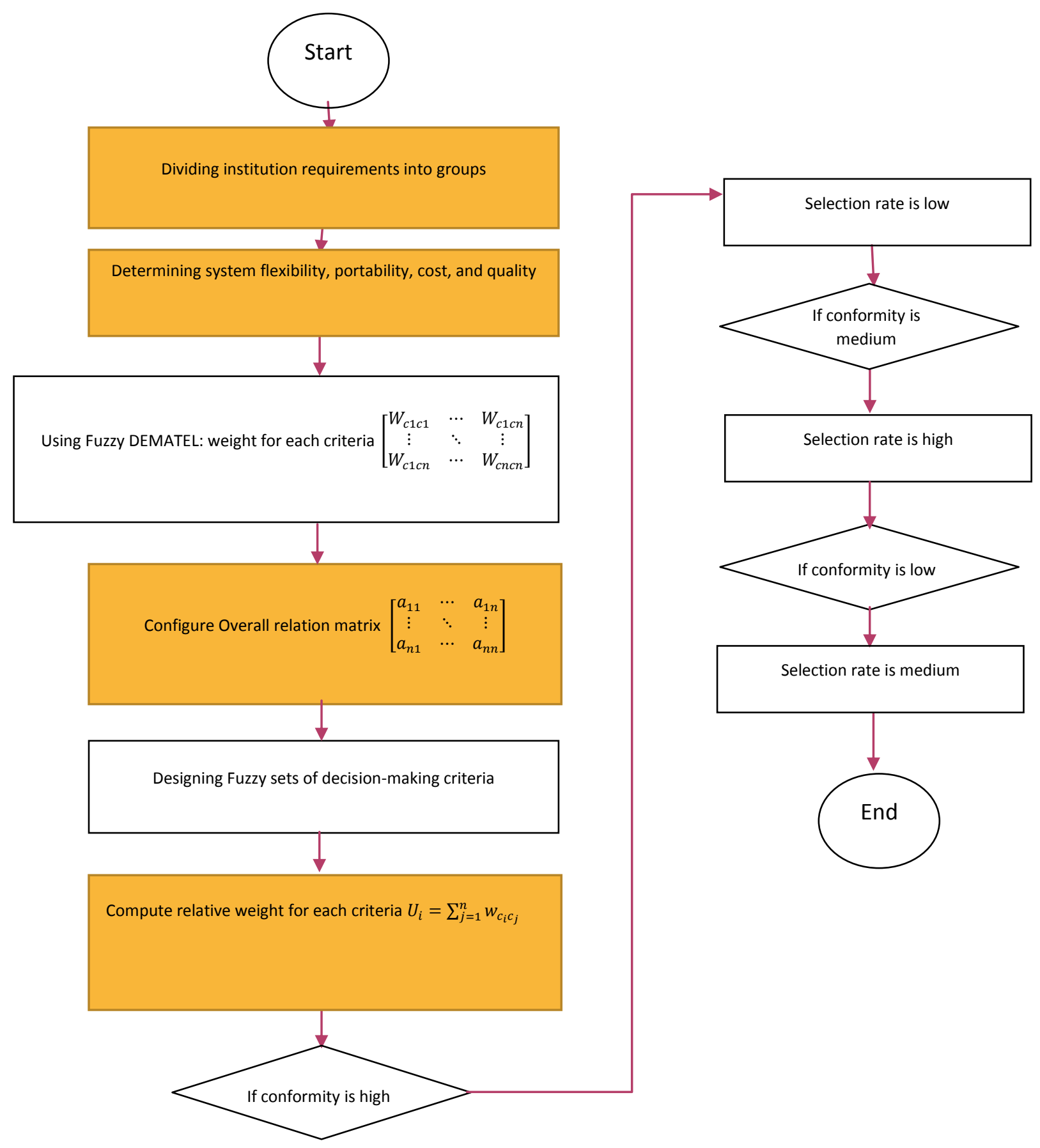

Figure 7: The Enhanced Proposed Algorithm to Select e-Learning System

Note: The Yellow Boxes Show The Enhancements. 
As it was mentioned above, the enhancements were inserted. However, the steps were:

1. Determining the criteria e-learning system flexibility, portability, cost and quality.

2. Using Fuzzy numbers to select an e-learning system by giving weight for each criteria.

3. Configure Overall relation matrix

$$
\left[\begin{array}{ccc}
a_{11} & \cdots & a_{1 n} \\
\vdots & \ddots & \vdots \\
a_{n 1} & \cdots & a_{n n}
\end{array}\right]
$$

4. Apply heuristic algorithm for each relation matrix of criteria.

5. Use fuzzy sets: low, medium, and high.

6. Configure the relation matrix to give weight for each criteria of the system:

$$
U_{i}=\sum_{j=1}^{n} w_{c_{i} c_{j}}
$$

After conducting the algorithm (fuzzification), it is important to conduct "defuzzification" by converting fuzzy numbers into numeral values by using the following equation:

$$
Z^{*}=\frac{\int \mu_{A}(z) \cdot z d z}{\int \mu_{A}(Z) d z}
$$

Then, ERP e-Learning systems are estimated based on the selection rate as shown in table 1 .

Table 1: Fuzzy Sets of Output Selection Rate

\begin{tabular}{|l|c|l|}
\hline Out put & Range & Fuzzy sets \\
\hline \multirow{3}{*}{ Selection rate } & $1-5$ & Low \\
\cline { 2 - 3 } & $4-9$ & Medium \\
\cline { 2 - 3 } & $8-12$ & High \\
\hline
\end{tabular}

\section{ALGORITHM VALIDATION AND DISCUSSION}

The ultimate goal of this system is to develop the enhanced proposed algorithm to best select of ERP selection criteria that match the requirement of the academic institutions. The output variable is a value from 1 to 12 that are: low, medium, and high. In this fuzzy e-Learning system, three output selection rates are defined for each criteria as follow: the selection rate is low when the conformity is high, which means that the difference between this and all other systems is little and small, and the excellence is low. The selection rate is high when the conformity is low, which means that the difference is medium, and the excellence is high. The selection rate is medium when the conformity is medium, which means that the difference is not too far from other systems and the excellence is medium.

In this paper, three types of evaluations that were used to evaluate the effectiveness of proposed Fuzzy model that were user interface, experts' evaluation, and case study.

\section{User Interface}

The design of the user interface is a key element in FDSS design. Information management systems are programs to increase information of existing data in a database, update, scan, display and link information to language models to become an integrated model of DSS. User testing method is considered as one of the most popular methods of usability methods. User testing method represents the relationship between tools and their beneficiaries, and it is a powerful tool that allows users to perform, manage and use tasks in the best way possible. This paper has considered interviewing user's sample consisting of five users (who used the system), to determine their views and sensitivities regarding e-Learning system. These interviews took place after the test had finished. The interview sample was made up of five users who never used with this system before. The main objective of interview is to verify that questions about performance standards have achieved or not. The users found that the e-Learning system was simple, usable and error-free. Also, they found that design was appropriate for the type of requirement provided by the institutions as well as they felt pleasure when using this system.

\section{Experts Evaluations}

Experts' evaluations help to determine the flexibility, quality, and portability of user interface design of e-Learning system. These criteria were evaluated during interviews with five experts using the theory of multiple intelligences. For the effectiveness of the system, most experts have seen that system is easy and fast to learn, cross-platform compatibility and robust as well as structured. As the experts stressed that DSS in finding solutions to a particular problem depended on an enterprise data warehouse (EDW) that was built from several sources in designated institution as system. The credibility of information system is considered utmost importance because the decisions made by the system based on this information and therefore the effectiveness of the system depend on the credibility of information. Furthermore, they illustrated that DSS has many benefits such as improving decision-making efficiency, supporting decision-making, encouraging learning or training, facilitating interpersonal communication, providing support to different administrative levels, increasing regulatory oversight by decision-maker, revealing new ways of thinking about problem space, encouraging exploration and discovery decision support tools, and providing support for sequential, multiple and interdependent decisions.

\section{Case Study}

A case study for ERP selection was used to evaluate the effectiveness of proposed Fuzzy algorithm that was conducted in the academic institutions. As the ERP system should take into account having the criteria: flexibility, portability, cost and quality that are required by the academic institutions. The enhanced proposed algorithm has been validated by SAP ERP experts, since SAP ERP has the ability to weight criteria in evaluating proposed algorithm followed by SAP ERP experts to determine the score of each criteria for the e-Learning systems as the score more than 70 is considered as valid. In this paper, six experts' opinions were used to evaluate the criteria of flexibility, portability, cost and quality for ERP elearning system. The results showed that the enhanced proposed algorithm has achieved average score of 82.18 on scale $0-100$, which means the validity of the enhanced proposed algorithm.

\section{CONCLUSIONS}

This algorithm-based procures may be modified according to the changes that may take place in the main procedures. After that, the procedures will be evaluated by assessing the overall performance of the system because the algorithm will be the 
main core in system performance. Finally, the proposed algorithm is flexible because it can be modified without affecting the main operations. This property is one of the maintainability attributes that aim to bear on the effort needed for making specified modifications. Also, efficiency is one the properties of this algorithm and it are a collection of attributes that bear on the relationship between the level of performance of the software and the amount of resources used. However, efficiency consists of two criteria: time behavior, and resource behavior. On the other hand, Fuzzy numbers are used for ranking the e-Learning systems and it may be modified.

\section{ACKNOWLEDGMENTS}

This paper consumed huge amount of work, research and dedication. Still, conducting this paper would not have been possible if I did not have a support of many individuals at our establishment Bisher and Kariman Consulting Est. in Amman -Jordan. Therefore I would like to extend our sincere gratitude to all of them.

\section{REFERENCES}

[1] Vahidi, J. and Salookolayi, D. (2014) 'A model for selecting an ERP system with triangular fuzzy numbers and mamdani inference', Journal of Mathematics and Computer Science, Vol.9.pp. 46-54.

[2] Wei, C. and Wang, M. (2004) 'A comprehensive framework for selecting an ERP system', International Journal of Project Management, Vol.22, PP: 161-169.

[3] Lee, M., Chang, J., Chen, J. (2011) 'An Entropy Decision Model for Selection of Enterprise Resource Planning System', International Journal of Computer Trends and Technology, Issue 2011.

[4] Huang, L. and Wu, Y. (2005) 'Applying Fuzzy analytic hierarchy process in managerial talent assessment model', International Journal of Technology Management, Vol.30, No.2.

[5] Hawly, D. and Johnson, J. (2000) 'Artificial Intelligence Network Systems: A Network tool for financial Decision Making', Financial Analysis Journal, Vol 46, No 6, PP. 63-72.

[6] Chan, W. and Tong, T. (2007) 'Multi-criteria material selections and end-of-life product strategy: grey relational analysis approach', Materials \& Design, Vol.28, PP.1539-1546.

[7] Naser, S., Masri, A., Sultan, Y. and Zaqout, I. (2011) 'A Prototype Decision Support System for Optimizing the Effectiveness of E-learning in Educational Institutions', International Journal of Data Mining, Vol.1, NO. 4.

[8] DeLone, W. and McLean, E. (2003) 'The DeLone and McLean Model of Information Systems Success: A Ten Year Update', Journal of Management Information Systems, Vol.19, 4, 9-30.

[9] Wang, Y.-S., Wang, H.-Y., \& Shee, D. Y. (2007). Measuring e-learning systems success in an organizational context: scale development and validation. Computers in Human Behavior, 23(4), 1792-1808.

[10] Herrington, A., Herrington, J., Oliver, R., Stoney, S. and Willis, J. (2001) 'Quality Guidelines for Online Courses: The Development of an Instrument to Audit Online
Units', Proceeding of ASCILITE, Melbourne, Australia 263-270, December 9-12, 2001.

[11] Steinberger, C. (2005) 'EduBITE: Developing an ELearning Environment for Enterprise-ResourcePlanning-Systems', in Proceedings of ICIHE03, Austria.

[12] Yang, R., Cowling, P. and Dahal, K. (2008) 'A Purchasing Decision Support System for a Rental Company', The 7th International Symposium on Operations Research and Its Applications (ISORA'08).

[13] Andrea, C. Mauro, G. (2012) Adoption of e-learning solution: selection criteria and recent trends, [PDF], University of Milan-Bicocca.

[14] Jeon, S. (2007) 'An integrated e-learning system for simulation-based instruction of anaesthesia machines', International Journal of Knowledge and Learning, Vol.3, No.1, pp. 106-120.

[15] Lasseter, M. and Rogers, M. (2004) 'Creating Flexible Elearning Through the Use of Learning Objects', Educause Quarterly, Vol.27, No.4, pp. 72-74.

[16] Browning, P. and Lowndes, M. (2001) JISC TechWatch Report: Content Management Systems, [pdf], UK, [online]: June 23, 2013, available at http://www.uwic.ac.uk/staffnet/is_forum/200102/Sub_Groups/Document_Management/Web\%20Conte nt\%20Management\%20Report_01-02.pdf.

[17] Chin, P., (2008) Developing a Content Management System Strategy, [e-Book], Publisher: Jupitermedia Corp, UK, [online]: 5 March 2013, available at: http://www.spidertg.com/cmsv5/images/stories/eBook_L ibarary/Developer/Developing $\% 20 \mathrm{a} \% 20 \mathrm{Content} \% 20 \mathrm{Ma}$ nagement $\% 20$ System\%20Strategy.pdf.

[18] Grover, S., Franz, P., Schneider, E. and Pea, R. (2012), The MOOC as Distributed Intelligence: Dimensions of a Framework \& Evaluation of MOOCs, Stanford Graduate School of Education, Stanford, CA. [online] http://lytics.stanford.edu/wordpress/wpcontent/uploads/2013/04/Framework-for-DesignEvaluation-of-MOOCs-Grover-Franz-SchneiderPea_final.pdf

[19] Schneider, E. (2013) 'Welcome to the moocspace: a proposed theory and taxonomy for massive open online courses., In the Proceedings of the Ist Workshop on Massive Open Online Courses at the 16th Annual Conference on Artificial Education.

[20] Kritikou, Y., Demestichas, P. and Adamopoulou, E. (2008) 'User Profile Modeling in the context of webbased learning management systems', Journal of Network and Computer Applications, Vol.31, pp. 603-627. Elsevier Ltd.

[21] Boag, P., (2010) Website Owner's Manual: the Secret to a successful Website, [PDF], Manning Publications Co., USA, [online]: 3 March 2013, available at: http://boagworld.com/uploads/WebsiteOwnersManual.pd f.

[22] Baylari, A. and Montazer, G. A. (2009) 'Design a personalized e-learning system based on item response theory and artificial neural network approach', Expert Systems with Applications, Vol 36, Issue 4, Pages 80138021. 
[23] Yarandi, M., Jahankhani, H. and Tawil, A. (2013) 'A personalized adaptive e-learning approach based on semantic web technology', Webology, Vol.10, N.2, December, 2013.

[24] Webster, J. and Hackley, P. (1997) 'Teaching Effectiveness in Technology-Mediated Distance Learning', Academy of Management Review, Vol.40, pp. 1282-1309.

[25] Australian Government Report, (2009) 'How organizations are using e-learning to support national training initiatives', [PDF], Department of Education, [Online].

[26] Xhafa, F., Caballe, S., Rustarazo, I. and Barolli, L. (2010) 'Implementing a Mobile Campus Using MLE Moodle', In P2P, Parallel, Grid, Cloud and Internet Computing (3PGCIC), 2010 International Conference on (pp. 207-214). IEEE.

[27] Ajzen, I. (1985) From Intentions to Actions: A Theory of Planned Behaviour, [PDF], Edited by Khul, J. and Beckmann,J. Springer-Verlag, and Berlin Heidlberg. New York Tokyo.

[28] Venkatesh, V., Morris, M. G., Davis, G. B., \& Davis, F. D. (2003). User acceptance of information technology: toward a unified view. MIS Quarterly, 27(3), 425-478

[29] Ajzen, I. and Fishbein, M. (1980) Understanding Attitudes and Predicting Social Behavior, Englewood Cliffs,NJ: Prentice-Hall, Inc.

[30] Al-Adwan, A., Al-Adwan, A. and Smedley, J. (2013) 'Exploring students acceptance of e-learning using Technology Acceptance Model in Jordanian universities', International Journal of Education and Development using Information and Communication Technology (IJEDICT), Vol. 9, Issue 2, pp. 4-18.

[31] Baxley, B., (2003) Making the Web Work: Designing Effective Web Applications Indianapolis, IN: New Riders Publishing, 2002.

[32] Stein, S. and Harman, B. (2000) 'Distance learning-the global challenge'. In Advanced Learning Technologies,
2000. IWALT 2000. Proceedings. International Workshop on (pp. 197-200). IEEE.

[33] Moodley, S. (2002) 'Inclusive Education: Challenges for Distance Learning, Policy and Practice', Academic Development Centre -Technikon South Africa, Johannesburg.Technologies, vol.4, no.2, pp.162, 174.

[34] Black, P. and Wiliam, D., (1998) 'Inside the Black Box: Raising Standards through Classroom Assessment', Phi Delta Kappan, vol. 80, no. 2, pp. 139-148.

[35] Amelung, M., Krieger, K. and Rosner, D. (2011) 'EAssessment as a Service', IEEE Transactions on Learning Technologies, vol.4, no.2, pp.162, 174.

[36] Munoz-Merino, P., Kloos, C. and Munoz-Organero, M., (2011) 'Enhancement of Student Learning Through the Use of a Hinting Computer e-Learning System and Comparison With Human Teachers', IEEE Transactions on Education, vol.54, no.1, pp.164,167.

[37] VanLehn, K., Lynch, C., Schulz, L., Shapiro, J. and Shelby, R. (2005) 'Andes physics tutoring system: Five years of evaluations', In Proceedings of the 12th international conference on Artificial Intelligence in Education Amsterdam, The Netherlands, 2005, pp. 678685.

[38] Guzma, E. and Conejo, R. (2005) 'Self-assessment in a feasible, adaptive Web based testing system', IEEE Transaction on Education, vol. 48, pp. 688-695.

[39] Martin, K. and Arroyo, I. (2004) 'AgentX: Using reinforcement learning to improve the effectiveness of intelligent tutoring systems', Intelligent Tutoring Systems, Lecture Notes in Computer Science, Volume 3220, pp: 564-572.

[40] Edmundson, A. (2007) Globalized E-learning Cultural Challenges, Idea Group Inc (IGI).

[41] Jahanshahi, H., Frahadzareh, B. and Fotuhi, H. (2013) 'A new algorithm for ERP system selection based on fuzzy DEMATEL Approach', Advances in Environmental Biology, Vol.7, No.9, pp. 2509-2521. 